\title{
Numerical simulations of central stellar velocity dispersion drops in disc galaxies
}

\author{
H. Wozniak ${ }^{1,3}$, F. Combes ${ }^{2}$, E. Emsellem ${ }^{3}$, and D. Friedli ${ }^{4,5}$ \\ ${ }^{1}$ Laboratoire d'Astrophysique de Marseille, 2 place Le Verrier, 13248 Marseille Cedex 4, France \\ 2 LERMA, Observatoire de Paris, 61 avenue de l'Observatoire, 75014 Paris, France \\ ${ }^{3}$ Centre de Recherche Astronomique de Lyon, 9 av. Charles André, 69561 Saint-Genis Laval Cedex, France \\ ${ }^{4}$ Observatoire de Genève, 1290 Sauverny, Switzerland \\ 5 Gymnase de Nyon, 1260 Nyon, Switzerland
}

Received 28 April 2003 / Accepted 30 June 2003

\begin{abstract}
We present an interpretation of the stellar velocity dispersion drops discovered in the nuclear regions of three barred galaxy hosts of active/starburst galactic nuclei (NGC 1097, NGC 1808 and NGC 5728) by Emsellem et al. (2001). Using selfconsistent $N$-body simulations including stars, gas and star formation, we show that young stars born in the nuclear regions from dynamically cold gas have a lower velocity dispersion than the older stellar population. The young population (less than $0.9 \mathrm{Gyr}$ old in our models), being more massive and brighter than the older one at near infrared wavelengths, fully dominates the observed line-of-sight kinematics of the circumnuclear regions.
\end{abstract}

Key words. galaxies: active - galaxies: kinematics and dynamics - galaxies: nuclei - galaxies: Seyfert - galaxies: evolution galaxies: spiral

\section{Introduction}

One of the aims of the DEBCA (Dynamics of Embedded Bars and the Connection with AGN) project is to characterize the kinematics of stars and gas in the few hundreds inner parsecs. We have up to now obtained long-slit ISAAC/VLT spectroscopy of three Seyfert galaxies with double bars (Emsellem et al. 2001, hereafter Paper I). In all three objects (NGC 1097, NGC 1808 and NGC 5728), the velocity dispersion displays a significant drop at the nucleus. In the present paper, the third of a series, we present and discuss an interpretation of the stellar velocity dispersion drops based on new self-consistent $N$-body models.

The frequency of such central velocity dispersion drops among the spiral galaxies is still unknown. At the time of our observations (Paper I), the only other known case was NGC 6503 observed by Bottema (1989). Since then several others objects has been found in the literature, not always double barred galaxies. For instance, NGC 3627, 4303, 4579 and 4725 were found in the data published by Héraudeau \& Simien (1998) and Héraudeau et al. (1999). NGC 4477, a SB0 without a priori any sign of active star formation, also displays a central dispersion velocity drop (Jarvis et al. 1988). However, NICMOS archive images of this object shows the presence of dust lanes near the nucleus that could be interpreted as some

Send offprint requests to: $\mathrm{H}$. Wozniak,

e-mail: herve.wozniak@obs.univ-lyon1.fr gas inflow. Finally, some elliptical galaxies also show this phenomenon (e.g. NGC 1404, Graham et al. 1998) although the cause could be different from disk galaxies.

To explain the dispersion drop in the centre of NGC 6503, Bottema \& Gerritsen (1997) had to assume a distinct cold $\left(\sigma_{0} \approx 21.5 \mathrm{~km} \mathrm{~s}^{-1}\right.$ ) and compact (core radius of $64 \mathrm{pc}$ ) isothermal nucleus in addition to the large scale stellar disc with the same mass-to-light ratio. Indeed, they failed to self-consistently reproduce the central dispersion drop with $N$-body realizations of pure stellar disc-halo models, whatever the initial conditions. Moreover, any additional cool central stellar disc heats up quickly through gravitational instabilities.

Another explanation is the existence of two counterrotating bars, as suggested by Friedli (1996). This hypothesis is not supported by our kinematical observations in any of the three cases studied in Paper I. Finally, using a simple model with damped orbits simulating the behaviour of the orbits near an inner Lindblad resonance, we showed (Paper I) that in no case it was possible to reproduce any central drop for the velocity dispersion.

We have thus performed new self-consistent $N$-body simulations including stars, gas and star formation to check the joint effect of the gas inflow and star formation in the central kpc. In Sect. 2 we briefly describe the codes used in this study, the setup of the initial conditions and the evolution of the runs. Section 3 is devoted to the analysis of the line-of-sight kinematical results while we discuss these results in Sect. 4. 


\section{Description of the simulations}

\subsection{The codes}

In the present section, we describe the techniques used to perform self-consistent simulations including stars, gas and star formation. In order to check for model and/or code dependent results, we have applied two significantly different schemes for the computation of the forces, and the star formation prescription.

\subsubsection{The polar FFT-SPH code}

We used PMSPHSF, the $N$-body code developed in Geneva. This code includes stars, gas and recipes to simulate star formation. The broad outlines of the code are the following: the gravitational forces are computed with a particle-mesh method using a 3D polar grid with $\left(N_{R}, N_{\phi}, N_{Z}\right)=(31,32,64)$ active cells (Pfenniger \& Friedli 1993), the hydrodynamics equations are solved using the SPH technique (Friedli \& Benz 1993) and the star formation process is based on Toomre's criterion for the radial instability of gaseous discs (Friedli \& Benz 1995). For the present work, the radiative cooling of the gas has been computed assuming a solar metallicity. Metallicity change of gas particles is computed assuming net yields given by Maeder (1992). At birth, star particles are created from gas, thus they have the same metallicity.

Several simulations were performed with different numbers of particles and various initial conditions. Since all the results are qualitatively similar and for the sake of clarity we will mainly deal with four runs (A, B, C and D) described below.

\subsubsection{The Cartesian FFT-sticky particle code}

We also performed two others 3D simulations (Runs E and F) with stars and gas, including star formation, using the FFT algorithm to solve the Poisson equation, but with a Cartesian grid, to check possible problems of the polar one. The FFT $N$-body code uses the James (1977) method to avoid the influence of Fourier images. The useful grid is $128 \times 128 \times 64$, corresponding to $32 \times 32 \times 16 \mathrm{kpc}$, corresponding to a cell size of $250 \mathrm{pc}$. This size is also the softening length of the Newtonian gravity.

When an extended dark matter component is added, to control the disk stability and the bar strength, it is with a spherical rigid potential, in a Plummer shape:

$\Phi_{\mathrm{DM}}(r)=-\frac{G M_{\mathrm{DM}}}{\sqrt{r^{2}+r_{\mathrm{DM}}^{2}}}$

with $M_{\mathrm{DM}}$ and $r_{\mathrm{DM}}$ the mass and characteristic radius of the dark matter component respectively.

The gas dissipation is represented by inelastic collisions between particles (see e.g. Combes \& Gerin 1985) and the rebound coefficient in total velocity vectors is 0.65 . Star formation is taken into account, following a generalised Schmidt law: the star formation rate is proportional to the volumic density to the power $n=1.2$, provided that the density is larger than $1 \mathrm{H}$-atom $\mathrm{cm}^{-3}$, i.e. the rate of gas mass transformed in
Table 1. Masses (in $10^{10} M_{\odot}$ ) of the various populations at $t=0,400$ and 905 Myr for Runs A and B.

\begin{tabular}{lrrrrrr}
\hline \hline & \multicolumn{3}{c}{ Whole simulation } & \multicolumn{3}{c}{ Central kpc } \\
Time (Myr): & 0 & 400 & 905 & 0 & 400 & 905 \\
& & & & & & \\
\hline Run A & & & & & & \\
Old & 11.0 & $\ldots$ & $\ldots$ & 0.94 & 1.41 & 1.73 \\
Gas & 1.1 & 0.71 & 0.54 & 0.06 & 0.01 & $2.3 \times 10^{-4}$ \\
New & 0.0 & 0.39 & 0.56 & 0 & 0.27 & 0.42 \\
Total & 12.1 & $\ldots$ & $\ldots$ & 1.00 & 1.69 & 2.15 \\
\hline Run B & & & & & & \\
Total & 12.1 & $\ldots$ & $\ldots$ & 1.22 & 1.55 & 1.74 \\
\hline
\end{tabular}

stars is $\mathrm{d} m=\mathrm{d} t C_{*} \rho^{1.2}$. To compute this rate, at regular intervals of $\mathrm{d} t=10 \mathrm{Myr}$, the gas density is averaged in $500 \mathrm{pc}$ cells, and the probability of the gas particles to be transformed into stars computed by

$P=\mathrm{d} m / M_{\text {cell }}$

for all particles in this cell, of mass $M_{\text {cell }}$. The new stars formed have exactly the same mass as all gas particles, slightly smaller than the old stellar particles. This simple scheme corresponds to an instantaneous recycling of matter, since the continuous mass-loss from recently formed stars is not followed. The rate of star formation is normalised so that in a quiescent galaxy (cf. Run E), the consumption time-scale is of the order of 2 Gyr. Gas metallicity changes are not computed with this code.

\subsection{Initial conditions}

For all our simulations, an initial stellar population is setup to reproduce a disc galaxy with an already formed bulge. These particles form what we call hereafter the "old population" as opposed to particles created during the evolution ("new population").

\subsubsection{Runs A, B, C and D}

For these four runs, the initial stellar positions and velocities for 500000 particles are drawn from a superposition of two axisymmetric Miyamoto-Nagai discs of mass respectively $10^{10}$ and $10^{11} M_{\odot}$, of scale lengths respectively 1 and $3.5 \mathrm{kpc}$ and common scale height of $0.5 \mathrm{kpc}$. The gaseous component is represented by 50000 particles for a total mass of $10^{10} M_{\odot}$ distributed in a disc of scale length $3 \mathrm{kpc}$. We did not include any dark halo as we only deal with the central region of the galaxies (e.g. less than $1 \mathrm{kpc}$ ). These initial conditions are almost similar to the model $C_{\text {sol }}$ of Friedli \& Benz (1995) apart from the higher number of particles.

In Table 1 we give the masses of the various components (old and new populations, gas) for the initial time and after an evolution of 400 and 905 Myr. As we are mainly interested in the kinematics inside the central kpc, we also computed 
Table 2. Initial conditions parameters for Runs E and F.

\begin{tabular}{|c|c|c|c|c|c|c|}
\hline Run & $\begin{array}{c}r_{\mathrm{b}} \\
\mathrm{kpc}\end{array}$ & $\begin{array}{c}M_{\mathrm{b}} \\
10^{10} M_{\odot} \\
\end{array}$ & $\begin{array}{c}r_{\mathrm{d}} \\
\mathrm{kpc}\end{array}$ & $\begin{array}{c}M_{\mathrm{d}} \\
10^{10} M_{\odot} \\
\end{array}$ & $\begin{array}{l}r_{\mathrm{DM}} \\
\mathrm{kpc}\end{array}$ & $\begin{array}{c}M_{\mathrm{DM}}{ }^{*} \\
10^{10} M_{\odot} \\
\end{array}$ \\
\hline Run $E$ & 1. & 0.45 & 3.5 & 4.5 & 10. & 8.1 \\
\hline Run $F$ & 1. & 1.2 & 3.5 & 12. & 10. & 0. \\
\hline
\end{tabular}

${ }^{*}$ Mass inside $12 \mathrm{kpc}$ radius.

the masses within a cylinder of a radius $1 \mathrm{kpc}$ centred on the nucleus.

\subsubsection{Runs $\mathrm{E}$ and $\mathrm{F}$}

For these two runs, the stellar component is represented by 305000 particles, and the gas component by 76000 . The stellar disk is initially a Kuzmin-Toomre disk of surface density

$\Sigma(r)=\Sigma_{0}\left(1+r^{2} / r_{\mathrm{d}}^{2}\right)^{-3 / 2}$

truncated at $12 \mathrm{kpc}$, with a mass $M_{\mathrm{d}}$. It is initially quite cold, with a Toomre $\mathrm{Q}$ parameter of 1 . The bulge is a Plummer sphere, with mass $M_{\mathrm{b}}$ and characteristic radius $r_{\mathrm{b}}$. The time step is 1 Myr. The parameters of the two runs described here are displayed in Table 2.

The gas component is initialised in an exponential disk of characteristic radius $r_{\mathrm{g}}=4 \mathrm{kpc}$, and truncated at $16 \mathrm{kpc}$. All gas particles have the same mass, and the total gas mass is initially $10^{10} M_{\odot}$.

\subsection{Evolution of the models}

\subsubsection{Run A}

After $905 \mathrm{Myr}$, the mass inside the central $\mathrm{kpc}$ has increased by $1.146 \times 10^{10} M_{\odot}$ which roughly doubles the total mass in this region. The first cause of this mass inflow is the overall reorganization of the stellar mass distribution under the influence of the bar instability. Indeed, the mass of the old population has increased by a factor 1.8 , which represents $69 \%$ of the additional mass. The remaining $31 \%$ are mainly due to the new population since most of the gas accumulated in the central region has been consumed by the star formation. The extra mass in the form of gas and new stars amounts to $3.56 \times 10^{9} M_{\odot}$ at $t=905$ Myr.

The global SFR is displayed in Fig. 1. Apart from the first SFR peak at $t=100 \mathrm{Myr}$ which is due to the progressive switch-on of the star formation in the code, three others SFR maxima occur around 300, 500 and $600 \mathrm{Myr}$. For $t \lesssim 300 \mathrm{Myr}$, the bar and a bi-symmetric spiral structure spontaneously form. The gravitational torques applied on the gas by the bar and the spiral arms create several regions of very high gas density in which star formation is ignited. The SFR reaches $30 M_{\odot} \mathrm{yr}^{-1}$. As shown in previous works (e.g. Martin \& Friedli 1997), the $\mathrm{SF}$ is not homogeneously distributed over the whole disc but is mainly concentrated along the bar major axis and along the spiral arms. Indeed, $3 / 4$ of the star formation occurs in the central region $\mathrm{kpc}$ of the galaxy. The next two peaks at $t \approx 500$

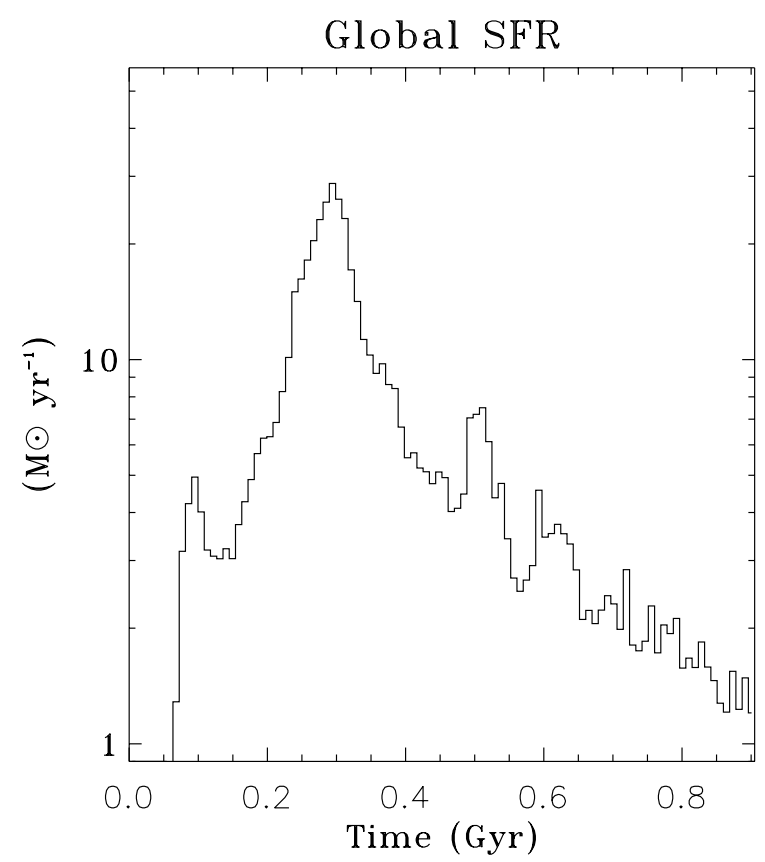

Fig. 1. Global star formation rate $\left(M_{\odot} \mathrm{yr}^{-1}\right)$ versus the elapsed time from the beginning of Run A. Star formation is inhibited during the first 58 Myr.

and $600 \mathrm{Myr}$ are the result of the gas inflow towards the central region of the disc. Such inflow is not stationary, but rather proceeds by burst.

\subsubsection{Runs B, C and D}

For the sake of comparison, we have also computed a pure $N$-body model (Run B), i.e. without gas and star formation. The total mass is the same mass than the whole components of Run A. This run tells us what is the evolution of the stellar mass and kinematical properties inside the central $\mathrm{kpc}$ in the absence of any dissipative component. As for Run A, the mass inside the central kpc increases but never reaches the same amount than in presence of gas and star formation. Bar length increases from $\approx 6$ to $\approx 8 \mathrm{kpc}$ between 400 and $905 \mathrm{Myr}$, longer than for Run A.

Another simulation (Run C) with twice the resolution of Run A has also been computed to check the effect of the smoothing parameter. For Run D gas is kept isothermal. We will bring up discussion on these runs in Sect. 4.

\subsubsection{Runs E and F}

Run E, stabilised by a rigid massive dark matter halo, develops only a very weak bar, and a spiral structure (Fig. 2); it is considered here as a control to investigate the bar influence. Run $\mathrm{F}$ has a strong bar, as can be seen in Fig. 3.

In both cases, the gravity torques due to the bar and spiral structure drive the gas inwards; this gas inflow is much more intense and occurs in a shorter time-scale in Run F. A nuclear gas disk is formed from the accumulation of gas in the centre, and new stars are actively formed there. 

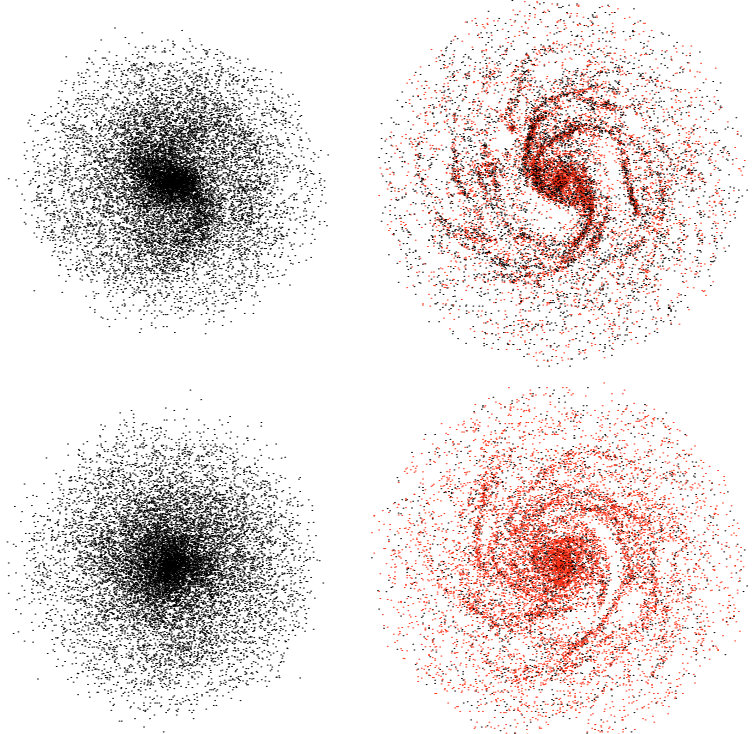

Fig. 2. Particle plots of the stellar (left) and gaseous (right) components, for Run E, at $t=1.2 \mathrm{Gyr}$ (top) and $2.4 \mathrm{Gyr}$ (bottom). The new stars are superposed in red to the gas. The scale is such that gas particules reach a radius of $16 \mathrm{kpc}$.

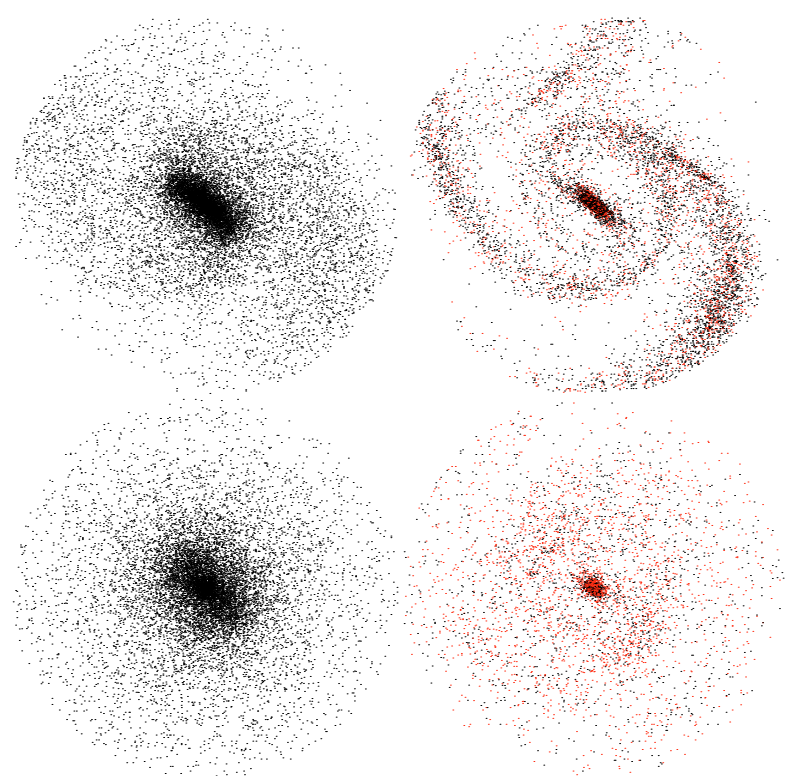

Fig. 3. Same as Fig. 2 for Run F, at $t=0.6 \mathrm{Gyr}$ (top) and $1.2 \mathrm{Gyr}$ (bottom).

\subsection{Mass-to-light ratio calibration}

The shape and equivalent width of absorption lines depend on the age and metallicity of the emitting stellar populations, especially for CO $2.3 \mu \mathrm{m}$ lines of very young stars (i.e. less than $10 \mathrm{Myr}$ old). The contribution of several stellar populations (or particles) must be integrated along a line-of-sight to obtain the observed velocity fields. However, in $N$-body simulations, the usual weighting by the mass of each population (or particle) is not representative of the real velocity field, since active star formation gives rise to a broad range of equivalent width,
Table 3. Geometrical parameters for the 3 galaxies. $\mathrm{PA}_{\text {disc }}, \mathrm{PA}_{\mathrm{pb}}$ (primary bar) and $\mathrm{PA}_{\mathrm{nb}}$ (nuclear bar) are measured from the North.

\begin{tabular}{lccrrrr}
\hline \hline Galaxy & $\begin{array}{c}\text { Dist. } \\
{[\mathrm{Mpc}]}\end{array}$ & $\begin{array}{c}\text { Scale } \\
{\left[{ }^{\prime \prime} \mathrm{kpc}^{-1}\right]}\end{array}$ & $\begin{array}{r}\mathrm{PA}_{\text {disc }} \\
{[\circ]}\end{array}$ & $\begin{array}{r}i \\
{[\circ]}\end{array}$ & $\begin{array}{r}\mathrm{PA}_{\mathrm{pb}} \\
{[\circ]}\end{array}$ & $\begin{array}{r}\mathrm{PA}_{\mathrm{nb}} \\
{[\circ]}\end{array}$ \\
\hline NGC 1097 & 16.8 & 12.3 & 130 & 37 & 138 & 30 \\
NGC 1808 & 10.9 & 18.9 & 133 & 70 & 143 & 157 \\
NGC 5728 & 37.0 & 5.6 & 30 & 55 & 33 & 79 \\
\hline
\end{tabular}

mass-to-light ratios, etc. However, the reconstruction of the $\mathrm{CO}$ lines shape and depth from simulations needs to sum up luminosity weighted and doppler shifted high resolution stellar spectra of various ages and metallicities. Due to the lack of a public library of high resolution spectra in the $K$ band covering a wide range of stellar ages and metallicities, this remains outside our scope. Thus, to check whether LOS kinematics could depend on the relative fraction of the young population versus the old one, we weight the LOS velocity contribution of each particle by its broad band luminosity. Kinematics thus could depend on the colour. We choose $V$ and $K$ bands since they respectively include $\mathrm{Mg}_{2}(517.5 \mathrm{~nm})$ and $\mathrm{CO}(2.3 \mu \mathrm{m})$ absorption lines.

For each stellar particle of Runs A, C and D, given its age and metallicity, mass-to-light ratio in the $K$ band was obtained by an interpolation through the tables of GISSEL99 (Bruzual \& Charlot 1993). The old population age and metallicity were assumed to be $10 \mathrm{Gyr}$ and solar. Obviously, this implicitly assumes that all old stars simultaneously born $10 \mathrm{Gyr}$ ago. However we found that our conclusions on the kinematical profiles are not sensitive to the assumption on the age and metallicity of the old stellar population (cf. Sect. 4). For Runs E and F, since we do not follow the gas metallicity evolution, we cannot calibrate the new population with the same rules. Thus, it has been calibrated assuming an $L / M 10$ times that of old particles.

\section{Line-of-sight kinematics}

Our main goal is to provide an interpretation of the LOS velocities observed with the ${ }^{12} \mathrm{CO}$ lines around $2.29 \mu \mathrm{m}$ (Paper I), especially the velocity dispersion drop within the central hundreds of pc around the active nucleus of NGC 1097, 1808 and 5728 .

To simulate the observations, we have rotated the positions and velocities of the particles so that the inclination angle and the projected position-angle between the bar and the disc major-axes match the observational values for the three objects (cf. Table 3 ). The masses were converted to $K$ luminosities and integrated along the LOS onto a grid to simulate a CCD camera. The grid angular resolution is identical to the spatial resolution of the observations. The velocities $\left(V_{\text {los }}\right)$ and velocity dispersions $\left(\sigma_{\text {los }}\right)$ were also integrated along the LOS, weighted by the luminosity of each particle. We extracted $V_{\text {los }}$ and $\sigma_{\text {los }}$ along a slit $00^{\prime} 6$ wide oriented along the bar main axes. We did not attempt to convolve the profiles with a PSF since we only planned to qualitatively reproduce the observations. We have also studied the new population contribution, separately 

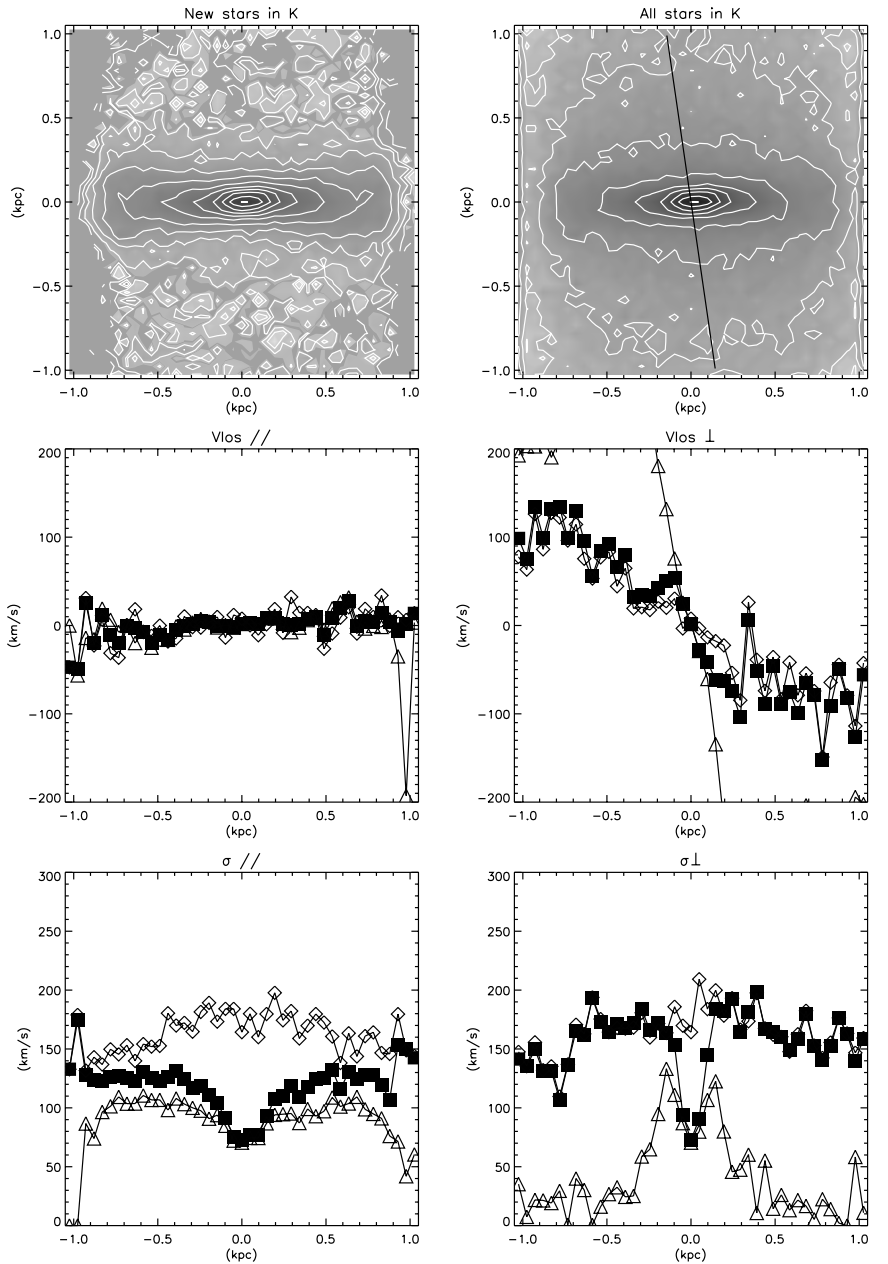

Fig. 4. LOS kinematical profiles for Run A at $t=400$ Myr. Projection parameters are those for NGC 1097. Top panels: $K$-band images (isophotes spaced by $1 \mathrm{mag}$ from 10 to $16 \mathrm{Kmag}^{\prime \prime 2}$ ) of the galaxy centre with the bar aligned along the $x$-axis (left panel: only stars born during the simulation, right panel: all stars). The line on the upper right panel indicates the position of the disc major-axis. Middle panels: LOS velocity profiles along the bar major-axis (left panel) and minor-axis (right panel). New stars velocities are represented by open triangles, old stars by open lozenge and the total luminosityweighted velocities by full squares. Bottom panels: LOS velocity dispersion profiles. The field of view is limited to the observational one (cf. Paper I).

from the old one. Since our conclusions will be qualitatively the same for the three objects, we will only display our results for the case of NGC 1097.

LOS velocities and velocity dispersions measured parallel and perpendicular to the bar in Run A are displayed in Figs. 4 and 5 respectively for $t=400$ and 905 Myr. Figure 6 shows the results obtained with Runs E and F computed with the cartesian code. Results for the run without gas and star formation (Run B) are shown in Fig. 7.

\subsection{Velocity curves}

Since our generic models do not perfectly match the global properties of the three galaxies (e.g. the total mass), we
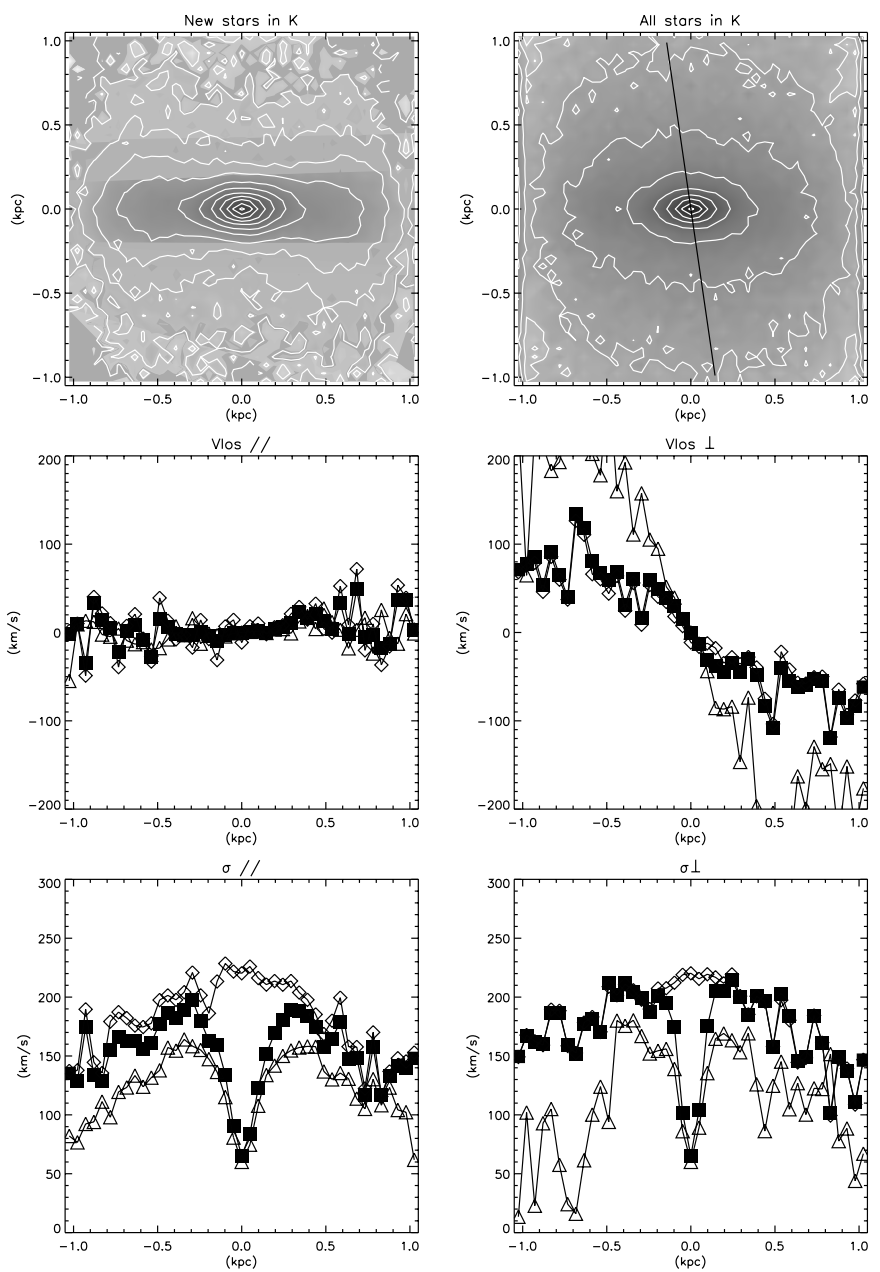

Fig. 5. As Fig. 4 for $t=905$ Myr.

cannot expect the $V_{\text {los }}$ curves will be a good approximation of the observed ones. For instance, models do not reproduce $V_{\text {los }}$ maxima inside the central kpc because of the lack of a nuclear embedded bar (cf. Sect. 4).

The $V_{\text {los }}$ profiles computed separately for old and new populations clearly show different kinematics, in particular along the bar minor-axis. The new population $V_{\text {los }}$ typically reaches $\approx 180-200 \mathrm{~km} \mathrm{~s}^{-1}$, at least twice the maximum value for the old population. The rough comparison of the pure $N$ body model $V_{\text {los }}$ (Fig. 7) with the old population $V_{\text {los }}$ shows some likeness.

\subsection{Velocity dispersion drops}

The ability of the models to qualitatively reproduce the central stellar $\sigma_{\text {los }}$ drops is obvious. The $\sigma_{\text {los }}$ drop is deeper for the model ( $\sigma_{\text {los }} \sim 50 \mathrm{~km} \mathrm{~s}^{-1}$ at the centre) than in our observations (between 90 and $150 \mathrm{~km} \mathrm{~s}^{-1}$ ). However, the central velocity dispersion is as low as $25 \mathrm{~km} \mathrm{~s}^{-1}$ in the case of NGC 6503 . Moreover, the model value is obtained in absence of any convolution with the seeing and/or the instrumental spectrograph profile. 

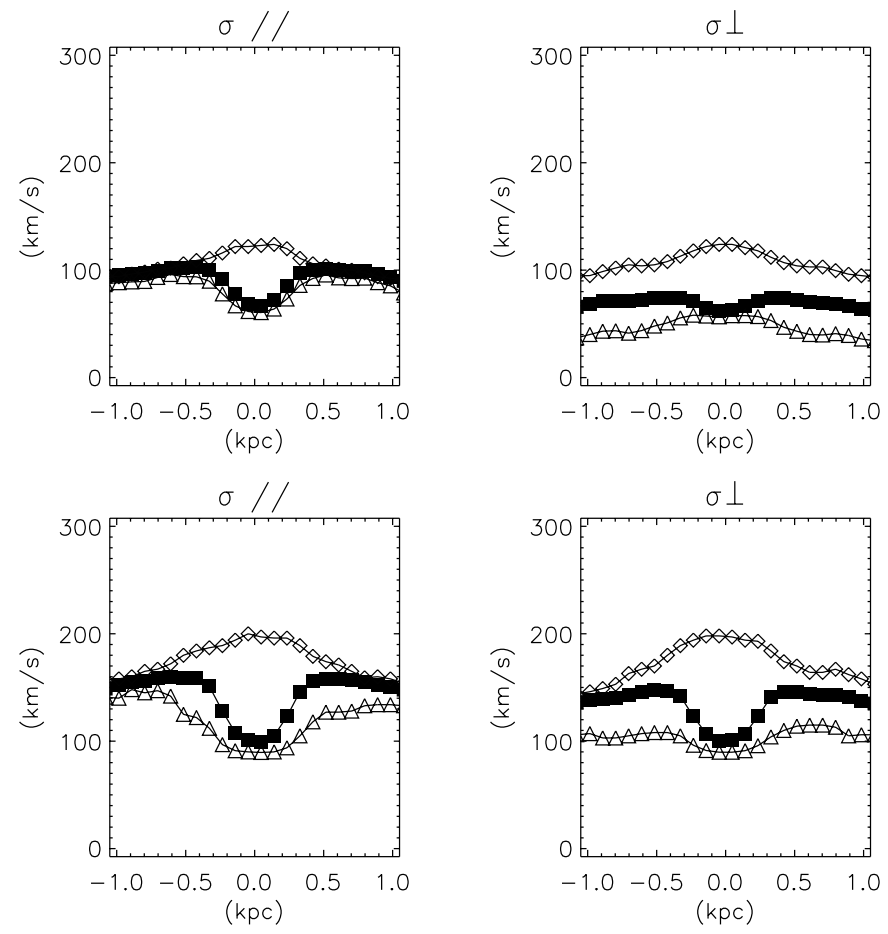

Fig. 6. Top panels: velocity dispersion profiles at $t=1.2 \mathrm{Gyr}$ for Run E. The three curves correspond to old stars (lozenge), new stars (triangles), and the total (full squares). The latter is luminosity weighted, with the new stars having an $L / M 10$ times that of old stars. Bottom panels: velocity dispersion profiles at $t=1.2$ Gyr for Run $\mathrm{F}$.

Run E (cf. Fig. 6) shows that the central velocity dispersion drop, although a weaker one, can form also without a strong bar.

A comparison between the $\sigma_{\text {los }}$ profiles along the two axes shows that the drop is more elongated along the bar major-axis than along the minor-axis as for real observations. This geometric property strongly suggests that the bar must play a leading role in setting up the velocity dispersion drop.

When the two stellar populations contributions are split it appears that only the new population is responsible for the central drop. On the opposite, the old population rather displays a small bump that is not visible in Run B (Fig. 7), the control run without gas. This suggests a gravitational heating of the initial stellar population due to the presence of the gas and new stars components.

Let us note that where the new population dominates the whole luminosity, $V_{\text {los }}$ of both populations are fairly similar. Thus, only $\sigma_{\text {los }}$ is useful to distinguish between new and old populations contribution.

\section{Discussion}

\subsection{Robustness}

A technical issue must be discussed in the first place: the effect of the softening parameter. Indeed, Fig. 8 shows unprojected kinematical properties of Run B without gas and star formation (thin line). This run also displays a very small velocity dispersion drop near the nucleus, in particular visible for the
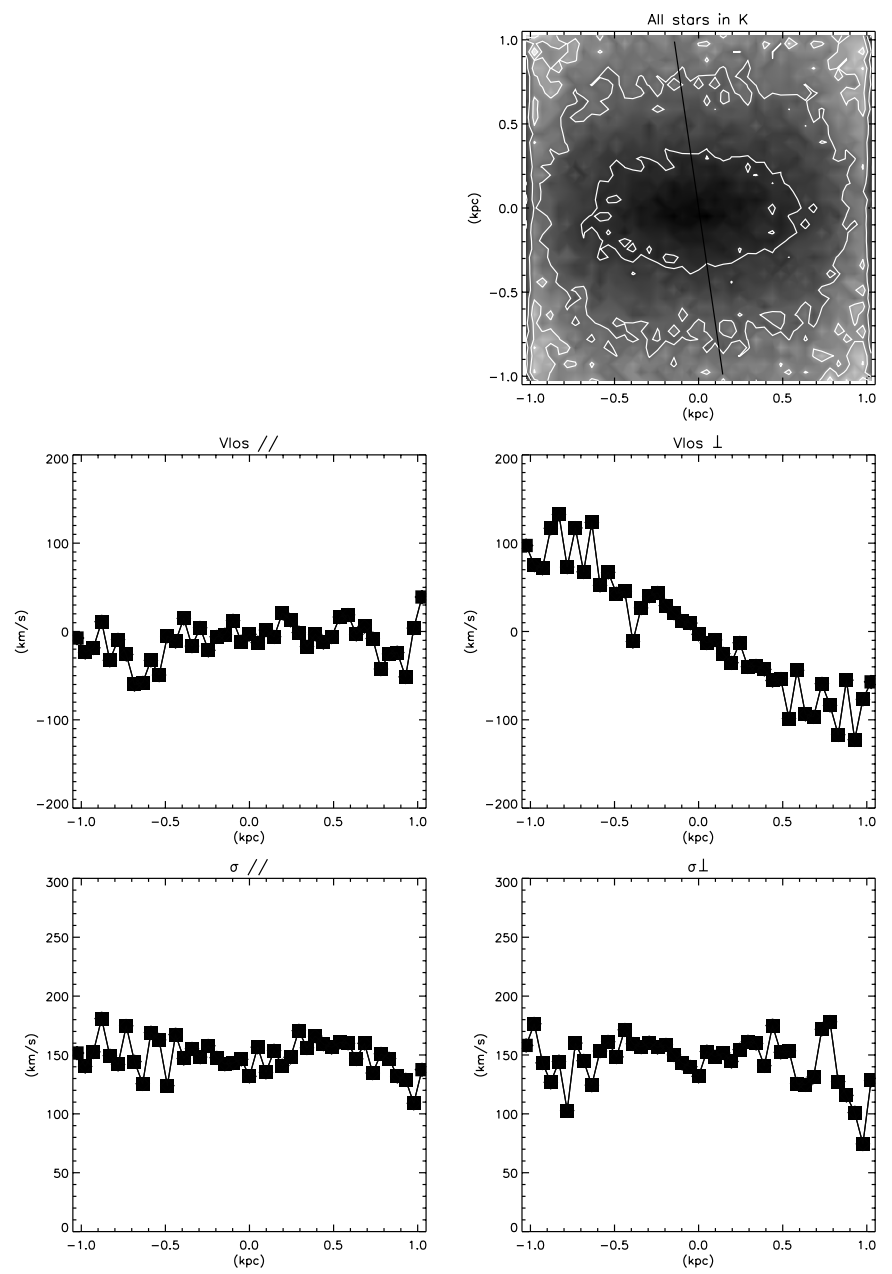

Fig. 7. Same as Fig. 4 but for Run B at $t=400 \mathrm{Myr}$, without gas and star formation.

tangential velocity dispersion. The amplitude of this drop is only $10-20 \mathrm{~km} \mathrm{~s}^{-1}$ at the most, far from the $50-70 \mathrm{~km} \mathrm{~s}^{-1}$ found for Run A. When $\sigma$ is integrated along the LOS, this drop becomes fairly unobservable. We impute this pure $N$-body effect to the softening parameter. To check the influence of the softening on our results, we have computed Run $C$ under the same conditions than Run A (i.e. stars, gas and SF) apart the grid resolution that has been doubled. The softening length has thus been halved with respect to Run A since the softening has been set to 1.1 times cell dimensions (cf. Pfenniger \& Friedli 1993). The unprojected velocity dispersion curves are displayed in Fig. 8 for $t=400$ Myr. At the first order, the nuclear drop is similar.

To check a possible effect by the radiative cooling and shock heating, the gaseous component has been kept isothermal for Run D. The unprojected stellar velocity dispersion profiles for this run is quite similar than for Run A. The maximum velocity dispersion difference between the two runs reaches $\approx 20 \mathrm{~km} \mathrm{~s}^{-1}$ but at a distance of $250 \mathrm{pc}$ from the centre. This does not lead to significant differences when kinematics is projected along the line-of-sight.

Our results are also robust with regard to the kind of $\mathrm{N}$-body code used to run the simulations. Indeed, Run E 


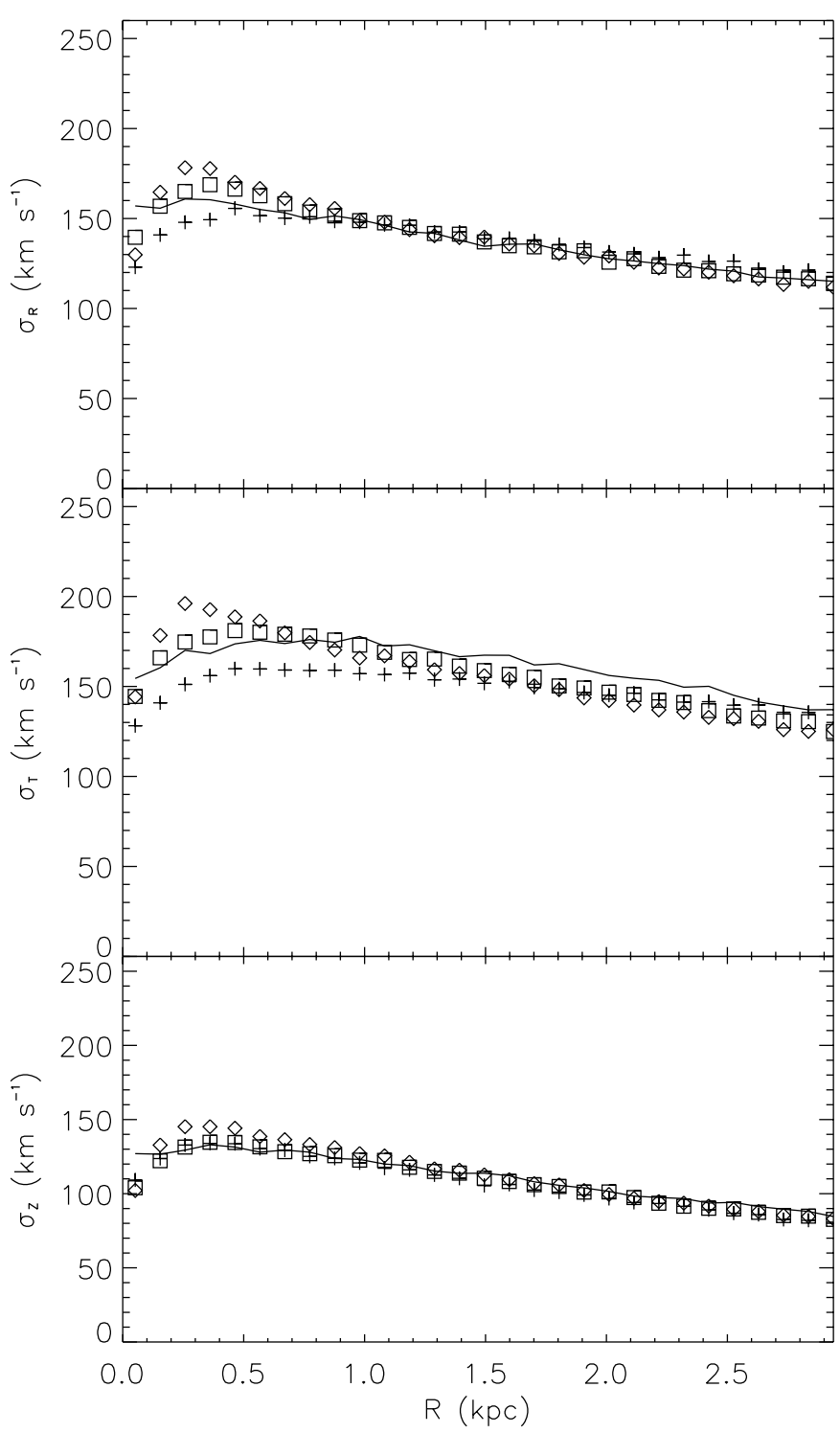

Fig. 8. From top to bottom : azimutally averaged radial, tangential and vertical unprojected stellar velocity dispersions for the pure stellar run (Run B, full line), for the full run (Run A, diamonds) and the control runs (Run C, crosses; Run D, squares), at $t=400 \mathrm{Myr}$.

and $\mathrm{F}$ show that the velocity dispersion drops do not depend on the modelling method (cartesian grid vs. polar grid, inelastic collisions vs. SPH).

In absence of any $M / L$ calibration, $\sigma_{\text {los }}$ is a mass-weighted instead of a luminosity-weighted mean of particles velocity dispersions. Even in this case, the drop is clearly present and can be easily observed. Thus it cannot be an artefact due to our photometric calibration. It cannot be either a polar grid artefact as the cartesian code confirms the effect. This dispersion drop is above all a dynamical phenomenon.

\subsection{Evolution of $\sigma$}

Let us now investigate further on which parameters the amplitude and radial extent of the drop depends on. For the sake of simplicity, we will temporarily assume that the new population is made of equal mass stars born at the same time (i.e. born from an instantaneous burst). Thus, $\sigma, M$ and $\Gamma$ being respectively the stellar LOS velocity dispersion, the mass and the mass-to-light ratio in the $K$ band, one can write the total LOS velocity dispersion at a particular location in a galaxy as:

$$
\sigma_{\operatorname{los}}^{2} \propto \frac{M_{\mathrm{o}}}{\Gamma_{\mathrm{o}}}\left(\sigma_{\mathrm{o}}^{2}+\frac{M_{\mathrm{n}}}{M_{\mathrm{o}}} \frac{\Gamma_{\mathrm{o}}}{\Gamma_{\mathrm{n}}} \sigma_{\mathrm{n}}^{2}\right)
$$

where the indexes "o" and " $n$ " indicate respectively the old and new populations. This implicitly assumes that both populations have the same mean LOS velocity which is roughly the case near to the nucleus. The contribution of the new population to $\sigma_{\text {los }}$ depends obviously on the mass ratio $M_{\mathrm{n}} / M_{\mathrm{o}}$ but also on the ratio of mass-to-light ratios $\Gamma_{\mathrm{o}} / \Gamma_{\mathrm{n}}$ since $\sigma_{\text {los }}$ is luminosityweighted. However, it is desirable to split the luminosity dependence into $M_{\mathrm{n}} / M_{\mathrm{o}}$ and $\Gamma_{\mathrm{o}} / \Gamma_{\mathrm{n}}$ contributions for our discussion.

In our $N$-body model, $M_{\mathrm{n}} / M_{\mathrm{o}}$ is self-consistently determined by the dynamical evolution and the star formation history of the galaxy. It cannot be a free parameter. Star formation increases $M_{\mathrm{n}}$ in the central region although variations in $M_{\mathrm{n}} / M_{\mathrm{o}}$ are not so easy to predict because of the simultaneous mass inflow of the old population (cf. Sect. 2.3). On the other hand, the evolution of $\Gamma_{\mathrm{o}} / \Gamma_{\mathrm{n}}$ depends mainly on populations ages. The wider the gap in age between the two populations the higher this ratio since $\Gamma$ increases with age. Consequently, when a young stellar population suddenly comes out, $M_{\mathrm{n}} / M_{\mathrm{o}}$ and $\Gamma_{\mathrm{o}} / \Gamma_{\mathrm{n}}$ simultaneously increase (i.e. $K$ luminosity increases), leading the old population contribution to become temporarily negligible in Eq. (1). When new stars age, $\Gamma_{\mathrm{o}} / \Gamma_{\mathrm{n}}$ diminishes since $\Gamma_{\mathrm{n}}$ increases faster than $\Gamma_{\mathrm{o}}$. Thus, in the simple case of an instantaneous burst of star formation, the velocity dispersion drop should be a transient feature.

As for the $M_{\mathrm{n}} / M_{\mathrm{o}}$ ratio, the new population spatial distribution is determined by the dynamical evolution. However, the observed spatial extent of the dispersion drop also depends on the $\Gamma_{\mathrm{o}} / \Gamma_{\mathrm{n}}$ ratio. Therefore, when the new population ages, it can be predicted that the drop extent becomes narrower.

Nevertheless, a real stellar system is made of old and new stars covering a wide range of ages, masses, mass-to-light ratios and velocities. Moreover, in circumnuclear regions, star formation mainly proceeds by bursts with the result that several generations of stars are superposed (cf. Kotilainen et al. 2000 for NGC 1097). Thus, it might be difficult to find any correlation between the velocity drop amplitude and/or width with the age of a nuclear starburst. For instance, a comparison of $\sigma_{\text {los }}$ along the bar major-axis between $t=400$ and 905 Myr (Figs. 4 and 5) clearly shows that the drop narrows while its amplitude increases. Such amplitude increase seems to be in contradiction with our above reasoning. In fact, looking only at the spatial distribution of the particles born between 805 and $905 \mathrm{Myr}$, we can see that the most recent star formation occurs in the central hundred parsec. In such innermost region, the mass surface density (top panels in Figs. 9 and 10) of the new population overtakes the old population one. This leads to a local $M_{\mathrm{n}} / M_{\mathrm{o}}$ increase but spatially restricted to the inner $100 \mathrm{pc}$. 

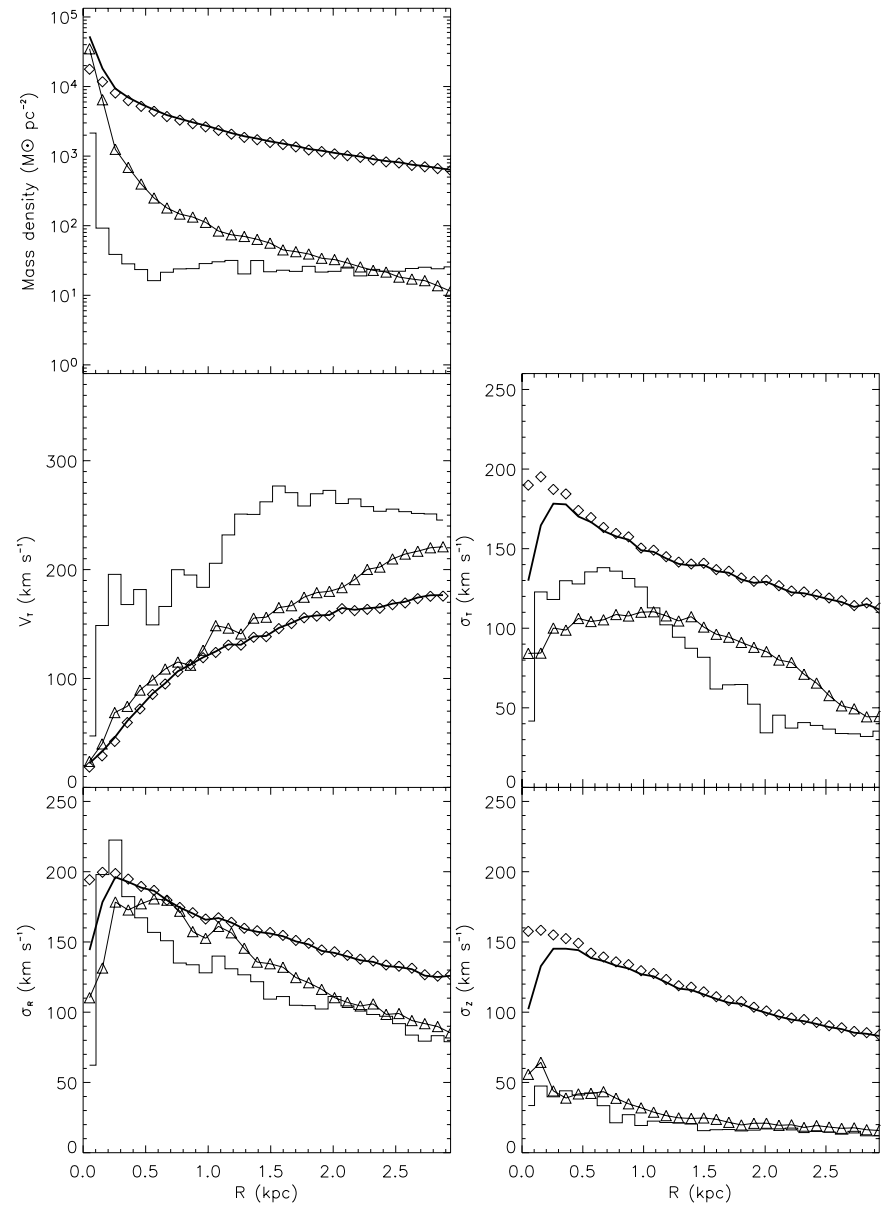

Fig. 9. Unprojected kinematics at $t=400 \mathrm{Myr}$ for Run A. Left panel, from top to bottom: mass density, tangential velocity and radial velocity dispersion. Right panels, tangential and vertical velocity dispersions. Open diamond are old stars and open triangles joined by a thin line are new stars. The sum of stellar contributions is plotted as a thick line. The gas is represented by histograms.

\subsection{Origin of low $\sigma$}

It remains to understand the origin of the low velocity dispersion of the new population. For this purpose, we display in Figs. 9 and 10 the tangential velocity and the radial, tangential and vertical velocity dispersions for both populations and for the gas. Obviously, these dynamical quantities should not be either photometrically calibrated or LOS integrated.

At $t=400 \mathrm{Myr}$, it is quite obvious that the velocity dispersion of the new population closely follows the gaseous one. Indeed, when new stars are created, they inherit the kinematical properties of their birthplace, in particular the velocity dispersion of the gas cloud. However, the gas velocity dispersion is lower than the old population because of the dissipative nature of the gas. This effect could be amplified in regions where dissipation is stronger, especially in the centre because the gas density is higher.

\subsection{Lifetime of $\sigma$-drop}

The new population velocity dispersions $\sigma_{\mathrm{R}}, \sigma_{\mathrm{Z}}$ and to a less extent $\sigma_{\mathrm{T}}$ increase between 400 and 905 Myr. This global
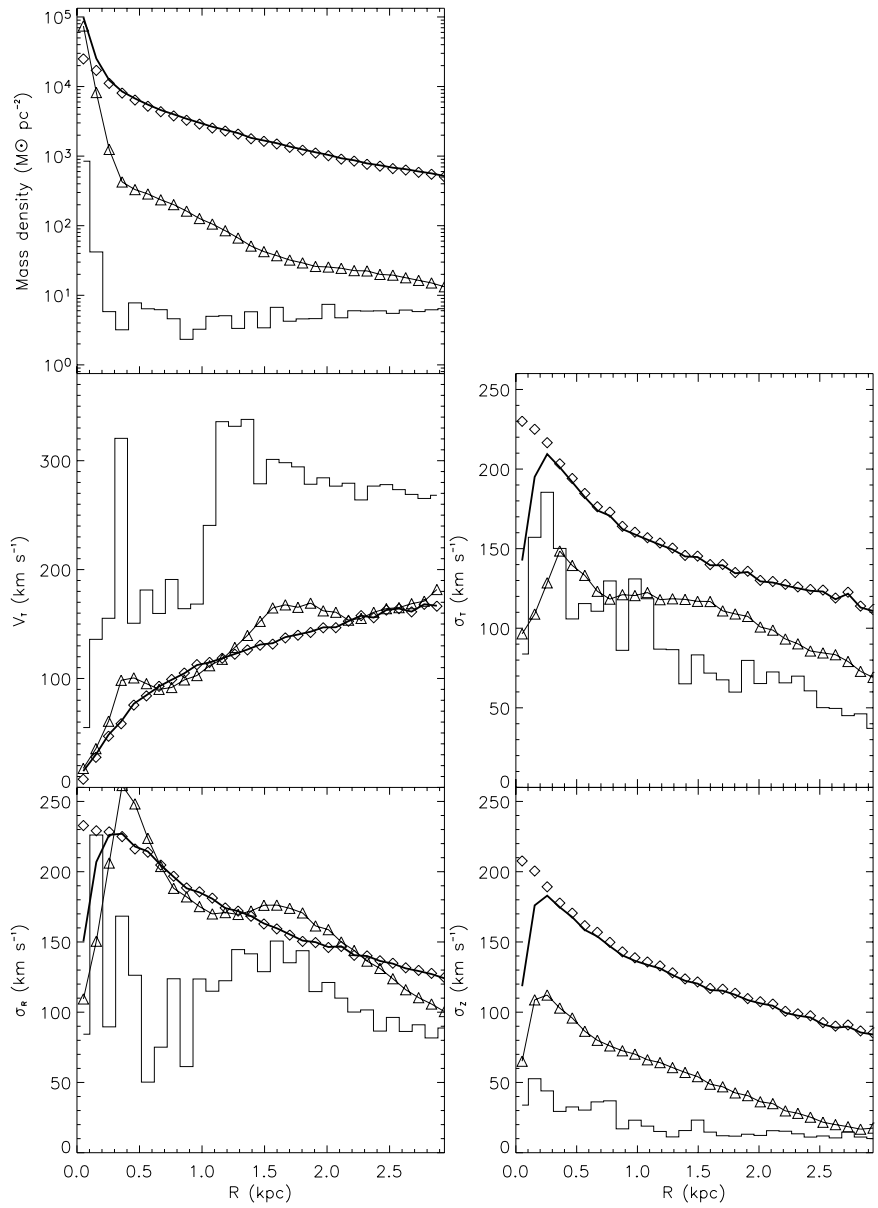

Fig. 10. As Fig. 9 for $t=905$ Myr.

heating of the new population is due to the gravitational interaction with the gas and the old and hotter population. This is not in contradiction with our interpretation of new stars low velocity dispersions as being a legacy of gas kinematical properties. Indeed, what we call "new population" contains the whole of the stars born from the beginning of the simulation. Since the global SFR decreases from 5.5 to $1.4 M_{\odot} \mathrm{yr}^{-1}$ between $t=400 \mathrm{Myr}$ and $t=905 \mathrm{Myr}$, this means that the genuine young stars, those with a low velocity dispersion, are less numerous at $t=905 \mathrm{Myr}$ than at $t=400 \mathrm{Myr}$. This heating is stronger in the central region where the bulge is growing up, especially in the $z$-direction.

On a short time scale, i.e. between $t=400 \mathrm{Myr}$ and $t=905 \mathrm{Myr}$ for Run A, $\sigma_{\mathrm{R}}$ and $\sigma_{\mathrm{T}}$ of the new population averaged inside the central kpc increase like a $t^{1 / 4}$ law. This is much slower than the $t^{1 / 2}$ law for a stellar disc (e.g. Jenkins \& Binney 1990 and references therein), partly because of the ongoing star formation which creates new stars with a low velocity dispersion. However, the kpc-averaged $\sigma_{\mathrm{Z}}$ doubles in the same time reflecting the fact that the drop shrinks while the surrounding region heats. This higher heating rate implies that, if star formation is switch off, $\sigma_{\mathrm{Z}}$ of the new population is expected to become similar to the old population $\sigma_{\mathrm{Z}}$ in at least 2 Gyr. However, the real lifetime of the drop also depends on the visibility of the $\mathrm{CO}$ lines at $2.3 \mu \mathrm{m}$. 
We did not try to carry on our simulations until the $\sigma$ drop disappears since it is questionable to use the close-box approximation on such a long-term evolution. Indeed, the accretion of gas from the outside should renew the material from which new generations of stars could from in the central region (Bournaud $\&$ Combes 2002). We thus postpone a direct study of the drop lifetime to a future paper.

\subsection{One or two bars?}

Finally, one should notice that our models display only one medium-scale bar (of length $\approx 3.5-4 \mathrm{kpc}$ in the case of Run $\mathrm{A}$ ) which remains roughly stable during a Gyr. There is no secondary nuclear bar embedded in the large scale one. We are thus, in principle, not able to study the kinematical decoupling of the nuclear bar, predicted by Friedli \& Martinet (1993) and observationally confirmed in Paper I. However, we have shown here that the velocity dispersion drop phenomenon can be independent of the presence of such embedded small scale bar. It is indeed directly linked to the ability of the bar to bring the gas towards the central region. We still need to study with future simulations of double barred galaxies whether the characteristics of the drops might be changed by the presence of a nuclear bar. This work is in progress.

\section{Conclusions}

We have performed $N$-body simulations with two independent codes including stars, gas and star formation. Our models are able to reproduce qualitatively the central velocity dispersion drops observed in Paper I, although these models do not develop a nuclear secondary stellar bar. Thus, they could also apply to other single barred objects.

We find that the stellar $\sigma_{\text {los }}$ drop is the kinematical signature of stars born from a dynamically cold gaseous component. The origin of the drop results from the conspiration of two factors. First, the new stars are concentrated towards the centre, and since they are just born from the low-dispersion gas component, their dispersion is much lower than for the old stars component. But second, the effect is amplified by the fact that the gas dispersion is also dropping towards the centre (and therefore the new stellar component too). This is due to the strong accumulation of gas towards the centre, in a nuclear disk, where dissipation is stronger than elsewhere, and where the gas is therefore cooling down efficiently.

Acknowledgements. Computations were partly performed on the Fujitsu NEC SX-5 hosted by IDRIS/CNRS. We are grateful to Dr. Rautiainen for his detailed reading of the manuscript.

\section{References}

Bottema, R. 1989, A\&A, 221, 236

Bottema, R., \& Gerritsen, J. P. E. 1997, MNRAS, 290, 585

Bournaud, F., \& Combes, F. 2002, A\&A, 392, 83

Bruzual, G., \& Charlot, S. 1993, ApJ, 405, 538

Combes, F., \& Gerin, M. 1985, A\&A, 150, 327

Emsellem, E., Greusard, D., Combes, F., et al. 2001, A\&A, 368, 52 (Paper I)

Friedli, D. 1996, A\&A, 312, 761

Friedli, D., \& Benz, W. 1993, A\&A, 268, 65

Friedli, D., \& Benz, W. 1995, A\&A, 301, 649

Friedli, D., \& Martinet, L. 1993, A\&A, 277, 27

Graham, A. W., Colless, M. M., Busarello, G., Zaggia, S., \& Longo, G. 1998, A\&AS, 133, 325

Héraudeau, P., \& Simien, F. 1998, A\&AS, 133, 317

Héraudeau, P., Simien, F., Maubon, G., \& Prugniel, P. 1999, A\&AS, 136,509

James, R. A. 1977, J. Comput. Phys. 25, 71

Jarvis, B. J., Dubath, P., Martinet, L., \& Bacon, R. 1988, A\&AS, 74, 513

Jenkins, A., \& Binney, J. J. 1990, MNRAS, 245, 305

Kotilainen, J. K., Reunanen, J., Laine, S., \& Ryder, S. D. 2000, A\&A, 353,834

Maeder, A. 1992, A\&A, 264, 105

Martin, P., \& Friedli, D. 1997, A\&A, 326, 449

Pfenniger, D., \& Friedli, D. 1993, A\&A, 270, 561 